\title{
The Role of Panels in Enhancing Legal Predictability
}

\author{
Douglas Glen Whitman
}

California State University, Northridge

18111 Nordhoff St., Northridge, CA 91330-8374

Phone: 818-677-4542 / Fax: 818-677-6264

glen.whitman@csun.edu

June 22, 2004 


\begin{abstract}
This article considers the justification for using panels of judges to make decisions in common law systems. The usual argument is that panels are more likely than lone judges to make correct judgments. This article suggests an additional justification: panels increase the predictability of law, so that potential litigants can anticipate correctly which legal rules will apply in their cases. Three models, each with a different conception of the legal process and the role of precedent, are employed to demonstrate the predictability-enhancing effect of panels. This effect is strongest when precedent has a substantial impact on how judges make decisions.
\end{abstract}

A prominent feature of many legal systems is the existence of courts - particularly at the appellate level - in which panels of judges make decisions. The justification for using a panel of judges rather than a lone judge seems obvious: a panel is more likely than a single judge to make a correct decision, where "correct" is understood to mean the dominant opinion in the legal community. This is true because the majority opinion of a group of judges is more likely than the opinion of a single judge to match the majority opinion of the larger population from which the judges are drawn - an insight widely known as the Condorcet jury theorem. ${ }^{1}$

Error correction is by far the most common argument in favor of having appellate courts, ${ }^{2}$ and the existence of multi-judge panels apparently adds to the perception that such courts are more likely to be trustworthy. As Judith Reznik (1984, 856) notes, "Appellate judges also lay claim to correctness through their numbers. ... Typically, the second [i.e., appellate level] judgment is a collective one, in which at least two appellate judges join. The intuition is that two thinkers are less likely to render poor decisions than is one." Of course, the judgment of what constitutes a poor decision has much to do with prevailing opinions in the legal community as a whole.

Steven Shavell $(1995)^{3}$ has made the error correction argument more precise, arguing that an appeals process can harness information litigants have about the correctness of decisions 
made in their cases. While it would be prohibitively expensive to demand a high level of accuracy in lower-level courts, a legal system can afford to invest greater resources in the accuracy of higher-level courts whose attention will be focused on the cases most likely to have been decided in error. What sort of investments would increase the accuracy (or correctness) of a court? One possible answer is to increase the number of judges. ${ }^{4}$ But as with other justifications from the perspective of error correction, Shavell's analysis depends on there being substantial agreement about what constitutes correctness in the legal system.

This article proposes another reason why panels might be desirable in a common law system: that a system employing such panels will exhibit greater predictability in the eyes of potential litigants than a system in which lone judges make decisions. If potential litigants cannot easily predict what rule a court will apply in their particular case, they will find it more difficult to choose their actions so as to avoid legal sanction and to coordinate their actions with each other. In other words, it is important for agents to be able to predict which rules the legal system will apply to them, regardless of whether those rules are deemed "correct." Panels have the capacity to enhance the predictability of the law, and this capacity is especially pronounced when precedent plays a large role in the legal system.

Part 1 extends Whitman's (2000) model of legal evolution to examine the certaintyenhancing properties of panels. In that model, judges balance their desire to announce legal rules with which they agree against their desire to protect their reputations. The original model, in which decisions were made by lone judges, derived conditions under which the common law process would be more likely to settle on a single legal rule (in a specific area of law) that potential litigants can rely on when making their decision, rather than oscillating between different legal rules. The present paper demonstrates that the incorporation of 
panels generally increases the likelihood of convergence on a single rule.

Part 2 explains, in briefer form, how including panel decisions would modify the results of two other models of legal evolution, thus indicating the robustness of the result derived in Part 1. The first of these models, taken from Priest (1977), incorporates a very weak role for precedent; the second, which relies on endogeneity of judicial preferences, gives precedent a much stronger role. The general conclusion is that panels almost always tend to increase legal predictability, but that tendency is most pronounced when precedent is strongest. Part 3 concludes by discussing applications, extensions, and opportunities for future research.

\section{Panels in a Model of Legal Evolution}

In this section, I will examine the effect of including three-judge panels in a reputation-driven model of judicial decision-making. This model was first introduced in Whitman (2000) to derive conditions under which a system of judge-made law can be expected to converge on a single legal rule for a class of disputes, rather than oscillating between rules. Here, the model is extended to show that replacing lone judges with panels weakens the conditions for convergence, thereby increasing the likelihood of converge on a single rule in a given area of law.

\subsection{Lone-Judge Decisions}

As this model has been fully described in Whitman (2000), a short recapitulation of the model's basic features will suffice here. The legal process is viewed as an endless sequence of cases arising, one at a time, in a particular area of law. Each time a case arises, a single judge is selected at random from the judge pool. This judge takes the rule announced by 
the previous judge as the legal precedent. The current judge must decide whether to follow precedent by announcing the same rule as the last judge, or to break precedent by announcing a different rule (which becomes the new precedent). ${ }^{5}$ Initially, there are only two available rules, dubbed Rule 1 and Rule $2 .{ }^{6}$

In deciding whether to follow or break precedent, a judge considers two factors. First, the judge has preferences over the available rules. Some fraction $\gamma$ of the judges prefer Rule 1 , and the remaining $(1-\gamma)$ of judges prefer Rule 2. The parameter $\gamma$ can be seen as a measure of division of opinion: when $\gamma$ is close to zero or one, division of opinion is low, and when $\gamma$ is close to one-half, division of opinion is high. For any judge $i$, the difference in utility from announcing the preferred over the unpreferred rule, known as the "preference satisfaction" value, ${ }^{7}$ is dubbed $v_{i}$. There is a known distribution of $v_{i}$ values in the judge pool, bounded below by $\underline{v}$ and above by $\bar{v}$.

Second, the judge must consider her reputation. If she follows the precedent, she takes no risk with her reputation, since following precedent is considered the norm. But if she breaks from precedent, she puts her reputation on the line. If the next judge follows the new rule, then the current judge experiences a gain in reputational utility of $u$. If the next judge rejects the new rule and returns to the old, the current judge experiences a loss in reputational utility of $d$. The judge makes her decision about whether to follow or break precedent on the basis of her total expected utility (from both preference satisfaction and reputational utility). For simplicity, assume that $\bar{v}<d$, which means that no judge will break precedent if she is certain to suffer a reputational loss from doing so.

A convergent equilibrium occurs when there is a legal rule that, once it becomes precedent, will never be displaced by the other. An oscillating equilibrium, on the other hand, 
occurs when the system goes back and forth between legal rules. Whitman (2000) derives a set of conditions for the existence of convergent equilibria, whose application depends on the relationship between division of opinion (as represented by $\gamma$ ) and judges' preference parameters. The conditions are most likely to be satisfied when division of opinion is relatively low.

\subsection{Panel Decisions}

I will now modify the lone-judge model to find analogous conditions for convergence under panel decisions. Each case is decided by a panel of three randomly selected judges instead of just one. The judges on a panel take the previous panel's decision as precedent and then vote on which rule to announce; the majority announcement constitutes the new precedent.

A few added assumptions are needed to define the model. First, I assume a single judge's reputation is affected only by the decision of the next panel, not by the decisions of the other members of her own panel. Second, it is necessary to specify how the reputation of a minority judge, whose decision was not the same as the panel's decision, is affected by the next panel's decision. For instance, if the precedent is Rule 1, and the panel votes two-to-one to break precedent in favor of Rule 2, how will the minority judge (known as the "squirrel") who voted for following precedent be affected by the next panel's decision? I will make two assumptions: (a) If the panel as a whole votes to break precedent, while the squirrel judge votes to follow precedent, then the squirrel judge's reputation will not be

affected by the next panel. Again, following precedent is taken as the norm. (b) If the panel as a whole votes to follow precedent, while the squirrel judge votes to break precedent, then the squirrel's reputation will be affected in the following manner: if the next panel rejects 
the current panel's majority decision, the squirrel gains $u$, but if the next panel affirms the current panel's majority decision, the squirrel loses $d$. A judge who votes with the majority will be affected just as in the single-judge model.

The decision of a panel is the outcome of a small three-person game, embedded within the larger game described by the system as a whole. Given that judges within a panel can, at least in many legal systems, consult with each other while making their individual decisions, it seems reasonable to treat the intra-panel game as a coalitional form game. This means that any outcome (panel decision) must be in the core of the game: if any coalition of judges can take actions that will thwart some outcome while making each member of the coalition better off, then that outcome will not occur. This treatment rules out certain implausible panel decisions. Suppose, for example, that all three judges are planning to follow precedent, even though two of the three would prefer to be part of a majority breaking from precedent. Without coalitions, this outcome could be sustained, because any single judge might be deterred from voting to break by the prospect of being the squirrel. But if coalitions are allowed, two judges could agree to change their plans at once, thus forming a majority for breaking.

The coalitional form of the game assures that a panel will vote to break precedent if and only if at least two of the three judges prefer being in a breaking majority to following precedent. If there are two or more judges who would prefer to be in a breaking majority, they can form a coalition to assure the panel breaks precedent. This coalition is stable because a defection by one of these judges would either shift the panel's decision to following precedent (if only two were in the majority), or else it would leave the breaking majority intact and make the defector a squirrel (if three were in the majority). Either way, the 
defector would be in a worse position than if she were in the breaking majority. If, on the other hand, fewer than two judges prefer being part of a breaking majority, then the two (or more) judges who favor following precedent can form a coalition to follow precedent. Again, the coalition will be stable because a defection by a judge in the majority would necessarily make that judge worse off.

With these initial assumptions in place, we can derive the conditions under which the legal process will lead to convergence on a single rule. The calculations are are omitted but available from the author on request. ${ }^{8}$ Here, I will contrast the results under panels with the results under single-judge decisions.

In order to have convergence on a single rule in the lone-judge model, there must be enough judges who support that rule to deter supporters of the other rule from breaking precedent. Suppose, for instance, that a large number of judges support Rule 1, and they are all willing to break from a precedent of Rule 2. If the number of Rule 1 supporters is large enough, the Rule 2 supporters will never risk breaking a precedent of Rule 1, for fear of suffering a reputational loss. In the panel-decision model, the logic is essentially the same. The difference is that it takes fewer supporters of Rule 1 to get the same amount of deterrence. This is because the likelihood of a panel supporting Rule 1 is greater than the probability of a lone judge supporting Rule 1, provided that 1-preferrers constitute more than half of the judge pool.

Mathematically, the necessary condition for convergence on Rule 1 in the lone-judge model is $\gamma>\bar{y}$, where $\bar{y}$ is shorthand for the expression $(\bar{v}+u) /(u+d)$. The analogous 
condition for convergence on Rule 1 with panel decisions is:

$$
3 \gamma^{2}-2 \gamma^{3}>\bar{y}
$$

This is true because $\gamma$ is the likelihood of a lone judge preferring Rule 1 , while $3 \gamma^{2}-2 \gamma^{3}$ is the likelihood of at least two out of three judges in a panel preferring Rule 1. It is easy to show that so long as $\gamma>1 / 2,3 \gamma^{2}-2 \gamma^{3}>\gamma$. As a result, satisfaction of the necessary condition for convergence on Rule 1 in the lone-judge model guarantees satisfaction of the equivalent condition in the panel-decisions model. Moreover, the fact that $3 \gamma^{2}-2 \gamma^{3}$ is strictly greater than $\gamma$ when $\gamma>1 / 2$ means that there are some values of $\gamma$ for which convergence is possible under panels, but impossible under lone judges.

Similarly, the necessary condition for convergence on Rule 2 in the single-judge model is $(1-\gamma)>\bar{y}$, and the analogous condition under panels is

$$
3(1-\gamma)^{2}-2(1-\gamma)^{3}>\bar{y}
$$

The result is the same: so long as $(1-\gamma)>1 / 2$, the condition for convergence on Rule 2 is weaker under panels than under single judges. Simply put, convergence on a single rule is more likely to be possible with panel decisions than with single judge decisions. ${ }^{9}$

A sufficient condition for convergence is required because it is possible to have multiple equilibria: in addition to the convergent equilibrium, there could also be an oscillating equilibrium. The sufficient conditions rule out multiple equilibria, thereby leaving only a convergent one. In the single-judge model, the sufficient condition for convergence on Rule 1 
is satisfied when the fraction of judges who support Rule 2 is small enough that even if all of them plan to break a precedent of Rule 1 in favor of 2, they still cannot deter any supporter of Rule 1 from breaking precedent. This guarantees that if convergence on Rule 1 is possible at all (i.e., the necessary condition is satisfied), it will certainly occur. Under panels, the result is similar, except that panels exaggerate the smallness of the minority faction. As a result, the number of supporters of Rule 2 could be somewhat larger and still fail to deter any supporters of Rule 1 from announcing their favored rule.

Mathematically, the sufficient condition for convergence on Rule 1 in the lone-judge model is $(1-\gamma)<\underline{y}$, where $\underline{y}$ is shorthand for $(\underline{v}+u) /(u+d)$. The analogous condition under panels is

$$
3(1-\gamma)^{2}-2(1-\gamma)^{3}<\underline{y}
$$

It is easily shown that $3(1-\gamma)^{2}-2(1-\gamma)^{3}<(1-\gamma)$ whenever $\gamma>1 / 2$. Just as with the necessary condition, the sufficient condition is more easily satisfied under panels than under single judges.

Similarly, the sufficient condition for convergence on Rule 2 under single judges is $\gamma<\underline{y}$, while the analogous condition under panels is

$$
3 \gamma^{2}-2 \gamma^{3}<\underline{y}
$$

Equivalent logic again shows that the sufficient condition is more easily satisfied under panels than under single judges. In short, convergence on a single rule is more likely to be guaranteed under panels than under single judges.

Qualitatively, panels produce the same results as single judges: Oscillation between rules 
Figure 1.1: Convergence Thresholds

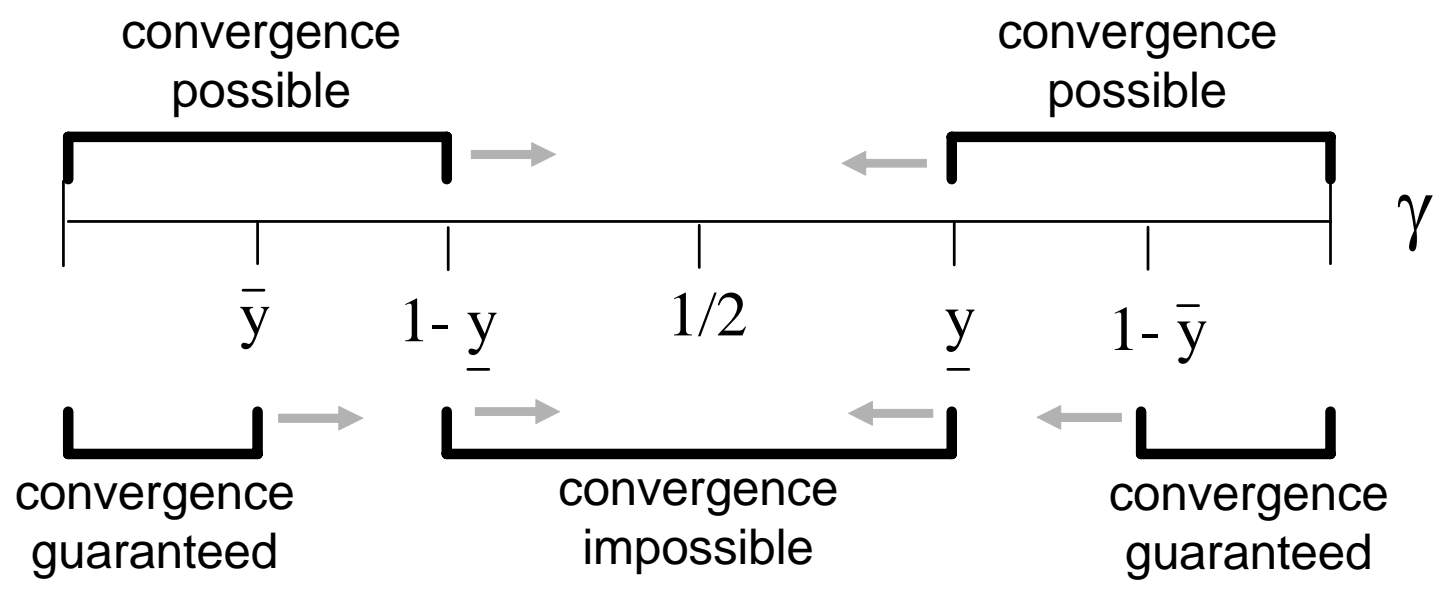

Thresholds shown are for single-judge model only. Arrows show direction in which panels alter thresholds.

is most likely when division of opinion is high (i.e., when $\gamma$ is close to $1 / 2$ ), whereas convergence is most likely when division of opinion is low (i.e., when $\gamma$ is close to zero or one). The difference between the two models is one of degree: panels shrink the range in which oscillation occurs and expand the range in which there is convergence. These results are summarized in Figure 1.1, which shows the threshold (necessary and sufficient) conditions for convergence under single judges. The arrows show how the use of panels moves these thresholds toward the center of the range, thereby expanding the regions in which convergence on a single rule can and will occur.

Whitman (2000) also shows that a "compromise" rule with certain properties can sometimes ameliorate a problem of oscillation between two rules. The legal system may converge on the compromise rather than oscillating between the original two rules, because the compromise acts as an "attractor" that pulls judges away from announcing their favored rules. A potential compromise rule is most likely to be viable when division of opinion is high - just 
when oscillation would be most likely to occur otherwise. It turns out that the same qualitative result holds under panels, but panels decrease the likelihood of such a compromise. The reason is that the viability of a compromise depends on there being an approximately equal division of power between the two factions of judges; but if either faction has a majority, panel decisions exaggerate its power. Thus, a narrow majority that would have been insufficient to thwart a compromise under single-judge decisions might be sufficient to do so under panel decisions.

\subsection{Implications}

The lone-judge version of the reputation-driven model derives both necessary and sufficient conditions for the convergence of the legal system on a single rule in a given area of law. Inserting panels into the model makes both sets of conditions easier to satisfy. There are some levels of division of opinion that will prevent convergence under lone judges, but that will allow convergence under panels. In this way, panels increase the predictability of the legal system by eliminating some situations in which the system would otherwise flip-flop between rules.

The predictability-enhancing effect of panels is limited to a world in which two rules compete for judges' attention. When a third ("compromise") rule is introduced, panels decrease the likelihood of the system being able to converge on the compromise relative to lone judges. The conclusion that panels enhance predictability must therefore be qualified. However, this does not pose a substantial problem for this paper's hypothesis. As observed earlier, convergence on a compromise rule is most likely to be possible when division of opinion is high - i.e., just when convergence is least likely in a world of two rules. While 
panels reduce the likelihood of convergence on a compromise, they also reduce the need for such a compromise because they expand the possibilities for convergence on one of the two initial rules.

\section{Alternative Models of Legal Evolution}

The model in the previous section relies on a specific conception of the legal process and the role of precedent. In this section, I will explain how inserting panels into two other models of legal evolution produces similar results, evidencing the robustness of the conclusions.

\subsection{The Priest Model}

Priest (1977) presents a model of legal evolution that purports to show that common law will tend to evolve toward efficient (i.e., wealth-maximizing) rules. I will modify Priest's model to show the effect of including panel decisions. Priest's model focuses on the efficiency of the legal system as a whole, asking what proportion of all legal rules in effect at a given time will be efficient. It does not address any particular area of law. It is possible, however, to tweak Priest's model so that it does deal with a single area of law. Modifying the model in this way allows an easier comparison between it and the other two models discussed in this article.

Suppose, again, that two legal rules vie for the attention of judges in a particular area of law. One rule is more efficient than the other. Whenever a case arises, the judge deciding the case will announce the efficient rule some fraction $a$ of the time, and the inefficient rule $(1-a)$ of the time. The parameter $a$ can be thought of as the fraction of judges in the judge pool who prefer the efficient rule. Priest's model is effectively "precedent-free" because of 
the constancy of the parameter $a$. The decisions of judges can always be characterized by use of this parameter, which is exogenous and unaffected by previous decisions. Nonetheless, in keeping with Priest's language, I will refer to the most recently announced rule as the precedent.

Priest argues that inefficient rules will be challenged in court more often than efficient rules. This is so because inefficient rules by definition create losses in wealth relative to efficient rules, and cases that involve higher stakes are less likely to be settled out of court. ${ }^{10}$ If $b$ is the likelihood of the efficient rule being challenged, and $c$ is the likelihood of the inefficient rule being challenged, then by the logic above, $b<c$. Given these parameters, it can be shown that the equilibrium (long run) fraction of time the efficient rule spends as precedent will be:

$$
X^{*}=\frac{a c}{a c+b(1-a)}
$$

The inefficient rule will prevail the remaining $\left(1-X^{*}\right)$ of the time. Examination of 2.1 reveals Priest's central result. Since $X^{*}>a$ whenever $b<c$, the fraction of time the efficient rule spends as precedent will exceed the fraction of judges who prefer the efficient rule.

If panels instead of lone judges make decisions, then the likelihood of the efficient rule being announced becomes $3 a^{2}-2 a^{3}$. This expression replaces $a$ in the calculation of the system's equilibrium, so that the new equilibrium is:

$$
X^{* *}=\frac{\left(3 a^{2}-2 a^{3}\right) c}{\left(3 a^{2}-2 a^{3}\right) c+b\left(1-\left(3 a^{2}-2 a^{3}\right)\right)}
$$

This equilibrium shares many properties of the lone-judge equilibrium. In particular, the equilibrium fraction of time that the efficient rule spends as precedent will be greater than 
$3 a^{2}-2 a^{3}$, the propensity of a single panel to announce an efficient rule. In this sense, the structure of the system still tends to favor more efficient rules.

But what effect do panels have on efficiency relative to the lone-judge model? It turns out that $X^{* *}>X^{*}$ if and only if $a>1 / 2$. If $a<1 / 2$, the panel equilibrium will cause the efficient rule to spend less time as precedent than the lone-judge equilibrium. In short, panels exaggerate the system's bias toward efficiency when individual judges favor efficiency more than half of the time, but they dampen the system's bias toward efficiency when individual judges favor efficiency less than half of the time. ${ }^{11}$

Panels also have an ambiguous effect on predictability, where predictability is defined as the distance from the equilibrium $\left(X^{*}\right.$ or $\left.X^{* *}\right)$ to either zero or one. In most cases, panels create greater predictability; but in the handful of cases where $a<1 / 2$ and $X^{*}>1 / 2$, panels can result in reduced predictability. Table 1 summarizes the results.

\section{Table 1: Panel Effects on Efficiency and}

Predictability in the Priest Model

\begin{tabular}{|c|c|c|}
\hline & $X^{*}>1 / 2$ & $X^{*}<1 / 2$ \\
\hline$a>1 / 2$ & $\uparrow$ efficiency; $\uparrow$ predictability & impossible \\
\hline$a<1 / 2$ & $\downarrow$ efficiency; ? predictability & $\downarrow$ efficiency; $\uparrow$ predictability \\
\hline
\end{tabular}

The ambiguity in the predictability effect of panels is attributable, I will contend, to the questionable assumption that $c>b$. This assumption was based on the idea that inefficient rules, because they generate higher stakes, will bring about more relitigation. Of course, such higher stakes will only occur if potential litigants' behavior is affected by whatever legal precedent is in place. But by the construction of the model, judges are not affected by legal 
precedent when they make their decisions. ${ }^{12}$ The probability of a judge choosing the efficient rule in a new case, $a$, is the same regardless of what rule the last judge announced in a similar case. Given that this is the case, there is no reason for agents to pay attention to what rule is officially the precedent in a given area of law; they will instead act on the expectation that in their own cases the efficient rule will be pronounced with probability $a$ and the inefficient rule with probability $(1-a)$. To put the point in a different way, consider agents making their behavior and litigation choices. Regardless of what rule was used to decide the last similar case, the agents will know (or be informed by their lawyers) that the rule used in this case will be one rule with probability $a$ and a different rule with probability $(1-a)$. Thus, the agents face the same incentives no matter what rule was announced most recently. For this reason, efficient rules may not be expected to be relitigated any more often than efficient ones in the context of Priest's model. This reasoning implies that $b=c$, not $b<c$.

Abandoning $c>b$ robs the model of its selection effect, eliminating the tendency toward efficiency. When $c=b$, the lone-judge equilibrium of 2.1 reduces to $X^{*}=a$, and the panel equilibrium of 2.2 reduces to $X^{* *}=3 a^{2}-2 a^{3}$. That is, the proportion of time the efficient rule spends as precedent is exactly equal to the likelihood of a single decisionmaker (lone judge or panel) picking that rule. Moreover, having $b=c$ means that panels unambiguously increase the predictability of the system. The special case where panels decrease predictability vanishes. ${ }^{13}$

There is another plausible assumption about relitigation rates that yields results intermediate between Priest's (strong tendency toward efficiency and ambiguous effect of panels) and mine (no tendency toward efficiency and unambiguous effect of panels). If the legal system is hierarchical in nature, then different assumptions can be made about the attitude 
toward precedent of judges at different levels. Suppose that district court judges always follow the rule last announced by a court of appeals, while appeals court judges decide according to their opinions as summarized by $a$. Only some fraction of all new cases will reach the appellate level, so the remainder will have final decisions based on the last rule announced. For that reason, potential litigants have good reason to look at the standing rule, not just the parameter $a$, when choosing their behavior. By Priest's reasoning, this means a larger proportion of inefficient than efficient rules will have new cases arise under them, and hence more inefficient rules will be challenged at the appellate level as well. We can interpret $b$ and $c$ as the rates of relitigation-and-appeal for efficient and inefficient rules, respectively, and assume once again that $c>b$. This new assumption appears to rejuvenate Priest's conclusion of a tendency toward efficiency.

But is it really true that potential litigants will have good reason to assume the standing precedent will be binding on them? Only if their cases will reach the legal system in the same period they choose their behavior. The farther in the future the legal case is likely to arise, the less realistic it is to assume that the current precedent will still hold at that time. If agents think their cases will arise immediately, they'll rely on the current precedent - and therefore $b$ and $c$ should differ. If agents think their cases will arise indefinitely far in the future, they'll ignore the current precedent and assume the efficient rule will be in place $X^{*}$ of the time and the inefficient rule $\left(1-X^{*}\right)$ of the time when their cases come to court and therefore $b$ and $c$ should not differ.

Realistically, agents will assume their cases will arise in an intermediate period of time. This means $b$ and $c$ should differ, but by less than they would if agents myopically assumed the current precedent would apply to their cases. The farther in the future one's case is 
expected to arrive in court, the less difference there will be in the rules' relitigation rates. Priest's tendency toward efficiency is maintained (in the lone-judge version of the model), but its magnitude is reduced. ${ }^{14}$

How does this approach affect predictability? As the gap between $b$ and $c$ shrinks, the range of values of $a$ for which predictability decreases shrinks as well, until eventually there are no such cases. The upshot is that panels are most likely to increase the legal system's predictability when the difference in relitigation rates between different rules is small. The smaller is this difference, the greater is the predictability impact of panels. When there is no difference between rules' relitigation rates at all, panels unambiguously increase the predictability of the legal system relative to lone judges.

\subsection{The Endogenous Preference Model}

Suppose again that we have a single, well-defined class of legal disputes. And again suppose there are two rules vying for dominance. Within the class of disputes, a large number of cases occur each period (as opposed to cases arriving one at a time, as in both prior models). Each judge who decides a case has a preference over the two rules, and their preferences directly determine their decisions on cases. However, the judges' preferences are endogenously determined by the disposition of cases in the previous period. If, for instance, Rule 1 were announced in 60 percent of cases in the last period, then 60 percent of judges prefer Rule 1 in the present period. Thus, precedent is treated as affecting judges' preferences directly, rather than mediating their preferences through reputation (as in the Whitman model) or not affecting them at all (as in the Priest model).

In this model, lone-judge decisions produce no inherent tendency toward convergence. 
Suppose that, to begin with, 60 percent of judges prefer Rule 1. If judges are randomly selected to decide cases, then Rule 1 will be announced in an expected 60 percent of cases this period, and therefore an expected 60 percent of judges will prefer Rule1 next period as well. Movement toward greater consensus on one rule or the other only occurs through random variation in the assignment of judges to cases. But inserting panels into the process creates movement toward convergence. If an initial 60 percent of judges favor Rule 1, and threejudge panels are selected randomly from the judge pool, then application of the Condorcet Jury Theorem shows that a panel will have a greater than 60 percent chance $(64.8$, to be precise) of announcing Rule 1. As a result, an expected 64.8 percent of judges will prefer Rule 1 the next period, leading to a yet higher fraction (about 71.6 percent) preferring Rule in the next period after that. Eventually, 100 percent of judges will employ Rule 1.

This model can be generalized so that the fraction of present cases decided according to Rule 1 need not translate exactly into the fraction of judges supporting Rule 1 in the next period. If judges are disproportionately exposed to previous decisions that were in the majority, as might happen in a legal culture that emphasizes consensus, the fraction of judges supporting a rule could exceed the fraction of cases so decided. If judges are disproportionately exposed to previous decisions that were in the minority, as might happen in a legal culture that values dissent and diversity of opinion, the fraction of judges supporting a rule could be less than the fraction of cases so decided. But so long as there is a positive relationship between the fraction of cases decided with a particular rule and the fraction of judges who come to support it, the qualitative results are much the same as above. ${ }^{15}$ When current decisions have an amplified effect on future judicial preferences, even lonejudge decisions produce a tendency toward convergence - but panels increase the speed with 
which such convergence is reached. When current decisions have a muted effect on future judicial preferences, not even panels can produce perfect certainty - but they still move the system toward greater consensus than would exist under lone-judge decisions.

\subsection{Comparing and Contrasting the Models}

The three models presented in this article resist attempts to relate them by (for instance) including one or two as special cases of the third. Still, it is possible to draw some connections among them. In all three, judges have preferences about legal rules. Also in all three, judges are presumed to have the ability to decide what rule to apply, so the courts in question must be precedent-setting courts (meaning, at least for Anglo-American legal systems, appellate courts). In this regard, the primary difference among the models is that the Whitman and Priest models assume that cases come one at a time, while the endogenous preferences model assumes that cases come in cohorts. Whether one assumption is more plausible than the other is, to this author, not obvious.

Finally, all three models assume (initially) that only two legal rules compete for the attention of judges. ${ }^{16}$ This assumption is questionable, as there exist many areas of law where three or more rules might be in contention, and in such areas the conclusions of this paper may be inapplicable. But there do exist significant disputes in the law that take the form of a tension between two rules, or at least two perspectives: strict liability versus negligence in the law of tort, damages versus specific performance in the law of contract, balancing versus coming-to-the-nuisance doctrine in the law of nuisance, and so forth. It is

in these areas of law that this paper's predictions are most relevant. Also, some areas of law where a multiplicity of rules are in play can be seen as a series of pairwise decisions; 
for instance, the damages remedy might prevail over specific performance for a class of contracts, but then a subsequent choice would have to be made between expectation and reliance damages.

It is in their treatment of the role of precedent that the models differ most. The strength of precedent is proportional to the degree of influence previous decisions have on future decisions. In the Priest model, the role of precedent is very weak: previous decisions never determine future decisions in any way. Instead, judges simply make decisions in line with their exogenous opinions. In the endogenous preferences model, the role of precedent is very strong: previous decisions completely determine future decisions, because previous decisions endogenously determine judges' opinions, and those opinions lead directly to future decisions. In the Whitman model, precedent has a moderate role: previous decisions partially determine future decisions, because even though judges have exogenous opinions, they also have an incentive (based on reputation) to follow previous decisions in some circumstances despite their personal opinions.

What is most intriguing about these models is that, despite their differences, they produce quite similar conclusions. Each one shows the capacity of panel decisions to increase the predictability of the legal system. Moreover, together and separately, they demonstrate that the effect of panels is most pronounced when precedent is strongest. When precedent was weakest (in the Priest model), the predictability effect of panels was little more than the Condorcet jury theorem. When precedent was strongest (in the endogenous preferences model), panels created a dynamic process that led to progressively greater predictability over time, and the process accelerated as precedent increased in strength. Finally, when precedent had an intermediate role (in the Whitman model), panels did not guarantee the 
predictability of the system, but they increased the range of circumstances where it was likely to occur.

\section{Summary}

\subsection{Extensions and Future Research}

In the context of three different models, each with a different conception of the legal process and the role of precedent, this article illustrates the capacity of panels to increase legal predictability. That capacity is especially powerful when precedent plays a large role in shaping the attitudes or choices of judges about what rules of law to announce.

Although the calculations are simplest when panels have only three members, the models in this paper can be extended to account for panels with more members. The larger is a panel, the more likely is the majority of the panel to match the majority of the judge population as a whole; this is the Condorcet jury theorem. Inserting larger panels into the models will not reverse, and in some cases will even amplify, their conclusions.

If the capacity of panels to enhance predictability is accepted as one justification for using them, then it stands to reason that panels should be used more often when there is a greater concern with constraining disparate opinions among judges. In general, higher level courts are entrusted with greater discretion, which raises the possibility that higher level courts will also be characterized by greater unpredictability as judges make decisions in line with their own opinions. Requiring panels in these higher level courts provides a means of reining in diverse opinions to make appellate level decisions more predictable. Meanwhile, since district courts are given less discretion, panels are less necessary at that level. 
In a different vein, the predictability-enhancing effect of panels can be employed whenever there is substantial disagreement - and corresponding uncertainty - on a specific question of law. This contention provides an added justification for the doctrine that an issue should be a matter of controversy among lower courts before it is considered by higher level courts. If the time and resources of the higher courts are limited, it makes sense to use them where they are most needed to resolve differences of opinion among judges at a lower level. Similarly, if substantial disagreement persists even among panels for a particular class of disputes, an appellate court may sit en banc to increase the panel size for a greater predictability effect. Sitting en banc would be costly to do in every single case, but it is more justifiable for the most contentious issues.

As noted earlier, the application of the models is limited by their focus on areas with only two or three rules under consideration. Expanding the models to account for a multiplicity of rules could be a fruitful area for further research. Incorporating the influence of hierarchical court systems more explicitly could also prove useful. Finally, it would be worthwhile to explore the question of which of the models here, or another model entirely, best represents the role of precedent in the legal system. Do the opinions of judges change in response to previous opinions, possibly through the process of legal education, or are legal opinions largely independent of the system's history? Do judges generally make decisions in line with their own beliefs, or does concern for reputation mute the effect of individual opinions? As the models here indicate, the answers to these questions will naturally influence the impact of panel decisions on the development of law. 


\subsection{Remarks}

The most common justification for having panels of judges make decisions in a legal system, particularly at the appellate level, comes from the perspective of error correction. It is intuitively plausible that a group of judges is more likely than a lone judge to arrive at a good decision. But this justification is dependent on the belief that the majority opinion of judges is the correct criterion for determining what is a good legal rule. If one takes a different criterion for correctness of legal rules, such as efficiency, panels can actually decrease the likelihood of a correct decision.

This paper points to an alternative justification for the use of panels. This justification is not a substitute for other justifications, but a complement that can explain why panels might be desirable even if the majority opinion among judges does not match one's standard of correctness. The argument is that panels increase the law's predictability, so that potential litigants stand a better chance of avoiding punishment and coordinating their activities with each other and the courts. This point brings to mind Milton Friedman's $(1982,27)$ observation that "in many cases, the existence of a well-specified and generally accepted definition of property is far more important than what the definition is." More generally, there is value to the existence of clear rules, even for those who find the selected rules disagreeable. Panels are one device a legal system can employ to promote clarity in the law. 


\section{Notes}

${ }^{1}$ Named after the French mathemetician and philosopher who first enunciated it. For discussion of the Condorcet jury theorem see, for example, Grofman and Feld (1988) and Grofman, Owen, and Feld (1983).

${ }^{2}$ Other arguments include "hopes ... of altering outcomes based on changed circumstances; of imbuing some decisions with more meaning by having them made repeatedly and sometimes by prestigious actors; of giving individuals a sense of having been fully and fairly heard," Reznik (1984, 855). For more on justifications for appellate courts, see Dalton (1985) and Wilner (1968).

${ }^{3}$ This is one of very few articles in the law and economics literature that addresses the justification for appeals courts in any depth. (Shavell concurs: "The law and economics literature does not include any articles on appeals, to my knowledge, but Richard A. Posner's Economic Analysis of Law (4th ed. 1992) text, at 584-87, contains a section on the subject (emphasizing the question whether appeals should be allowed during the course of a trial or only when it is complete).") Even Shavell's article does not directly address the use of judge panels at the appellate level. Kornhauser (1992a, 1992b) and Kornhauser and Sager (1993) address how panels should make decisions in cases involving multiple areas of contention.

${ }^{4}$ Shavell $(1995,381)$ mentions this possibility when he says, "Society enjoys the option, after all, of investing in more skilled trial court judges, of increasing the number of judges who hear each case, of lengthening trial proceedings to allow for more evidence and argument to be considered, and the like."

${ }^{5}$ For a similar model structure, see Miceli and Cosgel (1994).

${ }^{6}$ Later, a third rule is introduced to explore the possibility of legal compromise.

${ }^{7}$ If the judge announces the unpreferred rule, on the other hand, she experiences "preference falsification" - a term originated by Kuran (1990). Since $v_{i}$ is the difference in utility, it represents both preference satisfaction and preference falsification.

${ }^{8}$ In most respects, the calculations follow those in Whitman (2000), though they are complicated by the inclusion of panel decisions. 
${ }^{9}$ Convergence on one rule or the other is always possible when $\bar{y}<1 / 2$, under both lone-judge and panel decisions.

${ }^{10}$ This logic is not airtight, and Priest's model has been challenged on various grounds. I will not attempt, in this article, to catalogue the problems with this model; instead, I will focus only on the theoretical difficulty that is relevant in the context of panel decisions.

${ }^{11}$ In fact, panels will completely cancel out Priest's efficiency effect in some cases. For example, when $a=0.2, b=0.02$, and $c=0.04$, the panel equilibrium is $X^{* *} \approx 0.188$, which is less than $a$.

${ }^{12}$ This is true regardless of whether one uses Priest's original version or my reformulated version of it.

${ }^{13}$ Panels reduce predictability only if $a<1 / 2$ and $X^{*}>1 / 2$. But if $a<1 / 2$ and $X^{*}=a, X^{*}$ cannot be greater than $1 / 2$.

${ }^{14}$ To be more specific, if agents believe their case will arise in period $t$, they will calculate a probability distribution over the two possible rules at $t$, conditional on the current rule. The difference in the relitigation rates $b$ and $c$ will correspond to the difference between the distribution conditioned on the current rule being efficient and the distribution conditioned on the current rule being inefficient. As $t$ approaches infinity, both of these conditional distributions will approach the limiting distribution characterized by the probabilities $X^{*}$ and $1-X^{*}$. Thus, as $t$ rises, $b$ and $c$ should get closer to each other.

${ }^{15}$ Some ancillary assumptions on the transition function are required. Details of the mathematical model are available from the author on request.

${ }^{16}$ The Whitman model, however, considers the possibility of a third rule entering the fray. 


\section{References}

[1] Cooter, Robert, and Kornhauser, Lewis. 1980. "Can Litigation Improve the Law without the Help of Judges?" 9 Journal of Legal Studies 139.

[2] Dalton, H. L. 1985. "Taking the Right to Appeal (More or Less) Seriously." 75 Yale Law Journal 72-107.

[3] Friedman, Milton. 1982. Capitalism and Freedom. Chicago: University of Chicago Press.

[4] Goodman, John C. 1978. "An Economic Theory of the Evolution of the Common Law." 7 Journal of Legal Studies 393.

[5] Grofman, B., and Feld, S.L. 1988. "Rousseau's General Will: A Condorcet Perspective." 82 American Political Science Review 567.

[6] Grofman, B., Owen, G., and Feld, S.L. 1983. "Thirteen Theorems in Search of the Truth." 15 Theory and Decision 261.

[7] Kornhauser, Lewis A. 1992. "Modeling Collegial Courts I: Path-Dependence." 12 International Review of Law and Economics 169.

[8] Kornhauser, Lewis A. 1992. "Modeling Collegial Courts II: Legal Doctrine.” 8 Journal of Law, Economics and Organization 441.

[9] Kornhauser, Lewis A., and Sager, Lawrence G. 1993. "The One and the Many: Adjudication in Collegial Courts," 81 California Law Review 1.

[10] Kuran, Timur. 1990. "Private and Public Preferences." 6 Economics \& Philosophy 1. 
[11] Landes, William M., and Posner, Richard A. 1976. "Legal Precedent: A Theoretical and Empirical Analysis." 19 Journal of Law and Economics 249.

[12] Miceli, Thomas J., and Cosgel, Metin M. 1994. "Reputation and Judicial DecisionMaking," 23 Journal of Economic Behavior and Organizations 31.

[13] Posner, Richard A. 1998. Economic Analysis of Law. New York: Aspen Publishers, Inc.

[14] Priest, George L. 1977. "The Common Law and the Selection of Efficient Rules." 6 Journal of Legal Studies 65.

[15] Reznik, Judith. 1984. "Tiers." 57 Southern California Law Review 837, at 855.

[16] Rubin, Paul H. 1977. "Why Is the Common Law Efficient?" 6 Journal of Legal Studies 51.

[17] Shavell, Steven. 1995. "The Appeals Process as a Means of Error Correction." 24 Journal of Legal Studies 379.

[18] Wangenheim, Georg von. 1993. "The Evolution of Judge-Made Law." 13 International Review of Law and Economics 381.

[19] Whitman, Douglas G. 2000. "Evolution of the Common Law and the Emergence of Compromise." 29 Journal of Legal Studies 753.

[20] Wilner, I. 1968. "Civil Appeals: Are They Useful in the Administration of Justice?" 56 Georgetown Law Journal 417. 